\title{
Bioconversion of waste office paper to gluconic acid in a turbine blade reactor by the filamentous fungus Aspergillus niger
}

\author{
Yuko Ikeda $^{\mathrm{a}}$, Enock Y. Park ${ }^{\mathrm{a} \text {, Naoyuki Okuda }}{ }^{\mathrm{b}}$ \\ ${ }^{a}$ Laboratory of Biotechnology, Department of Applied Biological Chemistry, Faculty of \\ Agriculture, Shizuoka University, 836 Ohya, Shizuoka, 422-8529, Japan \\ ${ }^{\mathrm{b}}$ Technical Developments Department, Tsukishima Kikai Co., Ltd., 17-15, Tsukuda, 2-Chome, \\ Chuo-ku, Tokyo, 104-0051, Japan
}

\footnotetext{
* Corresponding author. Tel/fax: +81-54-238-4887.

E-mail address: yspark@agr.shizuoka.ac.jp (E.Y. Park)
} 


\begin{abstract}
Gluconic acid production was investigated using an enzymatic hydrolysate of waste office automation paper in a culture of Aspergillus niger. In repeated batch cultures using flasks, saccharified solution medium (SM) did not show any inhibitory effects on gluconic acid production compared to glucose medium (GM). The average gluconic acid yields were 92\% (SM) and 80\% (GM). In repeated batch cultures using SM in a turbine blade reactor (TBR), the gluconic acid yields were 60 (SM) and 67\% (GM) with 80-100 g/l of gluconic acid. When pure oxygen was supplied the production rate increased 4 times higher than when supplying air. Remarkable differences in the morphology of A. niger and dry cell weight between SM and GM was observed. The difference in morphology may have caused a reduction of oxygen transfer, resulting in a decrease in gluconic acid production rate in SM.

Key words: Recycle; Cellulase; Gluconic acid; Waste paper; Saccharification; Aspergillus niger
\end{abstract}




\section{Introduction}

Since the Industrial Revolution, people have benefited from petroleum resources. As a consequence, serious environmental problems have occurred such as air pollution, global warming and deforestation. Finding alternatives to petroleum products is thus an increasingly important objective for research. Recently, instead of using petroleum products, products utilizing biomass as raw materials have been developed for many markets. One of these, cellulolytic biomass, is known as a carbon neutral material because it does not increase the amount of carbon dioxide in the air.

Waste paper is one of the cellulolytic biomasses targeted to be recycled because it is a cause of environmental problems in Japan. Today in Japan about 30 million tons of paper is produced and consumed each year. The increased visibility of recycling has caused an increase of public awareness so that $66.1 \%$ of annual paper production is collected and $60.2 \%$ is reused (Terasawa, 2005). Although this recycling ratio is relatively high, it has not yet reached a satisfactory level. Excess paper that is not used by the Japanese market is exported to other countries. However, the price of paper has been rapidly decreasing and in this decade alone paper prices have been reduced by half. Moreover, when paper materials are recycled, they are usually turned into lower grade paper products; for example, conversions from office paper to magazine paper and from cardboard to sanitary products. With further recycling of paper, fiber length in the paper becomes shorter. Since the shortening of paper fibers decreases the quality of paper, the maximum ratio of paper-to-paper recycling is said to be $65 \%$. In addition, paper manufacturers are concerned about waste fibers which are not fit for recycling. This 
byproduct is disposed of by incineration or landfill without being reused. However, due to the shortage of new suitable disposal sites, environmental awareness, and the awareness of the greenhouse effect, these methods of disposal will be impossible in the near future. Finding alternative ways to recycle paper is an urgent necessity. One way of using waste paper is to decompose it to reducing sugars and to convert the sugars to value-added bioproducts; one such use of reducing sugars is fermentation into ethanol (Scott et al., 1994; Wayman et al., 1993).

Bioconversion of waste paper to L(+)-lactic acid by the filamentous fungus Rhizopus oryzae has been studied previously by some of the present authors. Although the lactic acid yield from waste office paper hydrolysate was similar to that of a glucose medium, the production rate was inhibited by either xylose derived from hemicellulose or unknown compounds originating from paper pulp (Park et al., 2004).

This paper suggests an alternative way of using waste paper by converting it to gluconic acid. Gluconic acid has been used as a food additive, in sterilization solution or bleach in food manufacturing factories, and as salt in chemical components for medication. Using waste paper hydrolysate, the gluconic acid yield and production rate were compared to those obtained with a glucose medium in a flask and bioreactor using the filamentous fungus Aspergillus niger.

\section{Materials and Methods}

\subsection{Enzyme hydrolysis}


Waste office automation (OA) paper was used as the experimental material because of its availability. OA paper refers to the papers used in plain paper copiers (PPC), and waste OA paper is also defined as printed PPC paper after printing in a printer or copy machine. Four hundred grams of waste OA paper were cut into small pieces by a standard office shredder to rectangles $2 \mathrm{~mm}$ wide and $1.5 \mathrm{~cm}$ long, and were used for enzymatic hydrolysis without any further pretreatments (Park et al., 2001). They were dissociated into smaller pieces in $1500 \mathrm{ml}$ of water using a blender for 1 minute. Hydrolysis was done by an addition of 5\% Acremonium cellulase AUSO 301 (1127 fpu/g, Meiji Seika, Co., Tokyo, Japan) by weight of the dried waste paper (Park et al., 2002). Hydrolysis was carried out at $45^{\circ} \mathrm{C}$ for 2-3 days with sporadic mixing. During hydrolysis, $\mathrm{pH}$ was adjusted to 4-5 with 2N HCl. After hydrolysis, the hydrolysate was separated from the slurry and immediately stored in a $-35^{\circ} \mathrm{C}$ freezer.

\subsection{Microorganism and medium}

The microorganism used in this study was Aspergillus niger IAM 2094, which consumes glucose as a carbon source and produces gluconic acid (Sakurai et al., 1989; Sankpal et al., 1999 and 2002). Spore formation was done on potato dextrose agar slants, and then the spores were stored in $\mathrm{a}-80^{\circ} \mathrm{C}$ freezer.

The preculture medium was composed (g/l) of glucose, 50; yeast extract, 3; malt extract, 3; polypeptone, 5; calcium carbonate, 20. The calcium carbonate was used to 
adjust $\mathrm{pH}$ to 5-6. Production medium was composed (g/l) of carbon source, 50 or 100; $\left(\mathrm{NH}_{4}\right)_{2} \mathrm{SO}_{4}, 1.35 ; \mathrm{Na}_{2} \mathrm{HPO}_{4}, 0.2 ; \mathrm{MgSO}_{4} \cdot 7 \mathrm{H}_{2} \mathrm{O}, 0.15$; calcium carbonate, 60 (Sakurai et al., 1989). However, when enzymatic hydrolysate was used as a carbon source, glucose concentration was adjusted to 50 or $100 \mathrm{~g} / \mathrm{l}$ with distilled water and then the ingredients of the production medium, except for glucose and $\left(\mathrm{NH}_{4}\right)_{2} \mathrm{SO}_{4}$, were added. The production medium containing enzymatic hydrolysate as a carbon source is defined as the saccharified solution medium (SM) and the production medium containing glucose as a carbon source as glucose medium (GM). All media and flasks were sterilized using an autoclave at $121^{\circ} \mathrm{C}$ for $20 \mathrm{~min}$.

\subsection{Gluconic acid production}

$2 \times 10^{6}$ spores/ml of A. niger IAM 2094 were inoculated into $500 \mathrm{ml}$ Erlenmeyer flasks containing $50 \mathrm{ml}$ of preculture medium $\left(10^{8}\right.$ spores/flask). The cultivation was carried out for $18 \mathrm{~h}$ at $30^{\circ} \mathrm{C}$ at 120 strokes per min (spm) in a reciprocal shaker. For the gluconic acid production, $5 \mathrm{ml}$ of preculture medium was inoculated into $500 \mathrm{ml}$ Erlenmeyer flasks containing $50 \mathrm{ml}$ of production medium. The cultivation was carried out at $30^{\circ} \mathrm{C}$ at $120 \mathrm{spm}$ in a reciprocal shaker for 72 hours. To investigate the contribution of reducing sugars contained in the enzymatic hydrolysate to the gluconic acid production, xylose and cellobiose were used as sole carbon source. Each experiment was carried out in three different flasks; the average was taken as data. The cultivation was carried out at $30^{\circ} \mathrm{C}$ at $120 \mathrm{spm}$ in a reciprocal shaker. 
The bioreactor, a turbine blade reactor (TBR) (2-1S, Sakura, Co., Ltd., Tokyo), was used for repeated batch culture of A. niger IAM 2094. The bioreactor was constructed of a glass cylinder with $800 \mathrm{ml}$ working volume, where the culture space (400 ml) was separated from the agitation space $(400 \mathrm{ml})$ by a stainless mesh cylinder and slit. $600 \mathrm{ml}$ of production medium was put into the bioreactor before autoclaving. The medium in the agitation space flowed upward along the wall of the bioreactor, passed through the culture space, and returned to the agitation space through the central stainless mesh. The whole system was sterilized by autoclaving at $121^{\circ} \mathrm{C}$ for $20 \mathrm{~min}$. The inoculum size was $10 \%$, and the cultivation was done at $30^{\circ} \mathrm{C}$. Either air or pure oxygen was supplied at $800 \mathrm{ml} / \mathrm{min}$ with agitation rate of 300-500 rpm.

Repeated batch cultures were carried out in a flask and the bioreactor. In the flask, the culture broth was centrifuged to separate supernatant and cell precipitate. Cell precipitate was returned to the flask and $50 \mathrm{ml}$ of fresh medium was then added. In the case of the bioreactor, the old medium in the agitation space was pumped out and a fresh one was pumped in using a micro tube pump (MP-3, Tokyo Rikakikai. Co. Ltd., Japan) when the carbon source concentration was lower than $10 \mathrm{~g} / \mathrm{l}$ in the culture. Each experiment was performed in triplicate; the average was taken as data.

\subsection{Analytical methods}

Biomass concentration was obtained by the dry cell weight method. The culture broth was filtered through Whatman No. 2 filter paper and the collected solids were 
washed twice with distilled water then dried at $80^{\circ} \mathrm{C}$ for $12 \mathrm{~h}$.

Glucose concentration was measured using a biochemistry analyzer (2700, YSI Inc., Yellow Springs, Ohio, USA). Xylose, cellobiose and other reducing sugars were analyzed using a HPLC (PU-980, JASCO, Co. Ltd., Japan) with Shim-pack CLC-NH column (Shimadzu, Kyoto, Japan). As a mobile phase, 75\% acetonitrile solution was used at a flow rate of $2 \mathrm{ml} / \mathrm{min}$. Detection was carried out by an refractive index detector (RI-930, JASCO, Co. Ltd., Tokyo).

Gluconic acid concentration was analyzed as follows: $1 \mathrm{ml}$ of sample and $1 \mathrm{ml}$ of fresh broth without calcium carbonate were mixed; $4 \mathrm{ml}$ of $0.1 \mathrm{M}\left(\mathrm{NH}_{4}\right)_{2} \mathrm{C}_{2} \mathrm{O}_{4} \cdot \mathrm{H}_{2} \mathrm{O}$ and $2 \mathrm{ml}$ of $1 / 50 \mathrm{~N} \mathrm{NH}_{4} \mathrm{OH}$ were added, and then the mixture was boiled for $10 \mathrm{~min}$. After cooling to room temperature, the supernatant was removed by centrifugation at $112 \mathrm{~g}$ for $5 \mathrm{~min}$. The resulting precipitate was washed with $5 \mathrm{ml}$ of distilled water three times, and then dissolved by heating in the presence of $6 \mathrm{ml}$ of $2 \mathrm{~N} \mathrm{H}_{2} \mathrm{SO}_{4}$. The final solution was titrated with $1 / 20 \mathrm{~N} \mathrm{KMnO}_{4}$.

Mycelial morphology in the TBR was analyzed using a scanning electron microscope (JSM-T300, JEOL, Ltd., Tokyo, Japan).

\section{Results}

\subsection{Gluconic acid production using SM in flask}

When the initial glucose concentration was adjusted to $50 \mathrm{~g} / \mathrm{l}$ in SM, $46.0 \mathrm{~g} / \mathrm{l}$ of 
gluconic acid was produced, while $40.4 \mathrm{~g} / \mathrm{l}$ of gluconic acid production was obtained from $50 \mathrm{~g} / \mathrm{l}$ of glucose in GM (Figure 1). The profiles of glucose consumption in SM and GM overlapped (Figure 1a). The yields of gluconic acid based on glucose consumption were $92 \%$ in the SM and $80 \%$ in the GM.

Reducing sugars, such as xylose and cellobiose containing in the culture broth of $A$. niger, were measured as shown in Figure 1b. The initial cellobiose (6.7 g/l) was consumed as quickly as glucose. On the other hand, $4.9 \mathrm{~g} / \mathrm{l}$ of xylose in SM was consumed for 72 hours by A. niger, but $43 \%$ of the initial xylose remained. The consumption rate of cellobiose was approximately four times higher than that of xylose. When xylose and cellobiose were used as the sole carbon sources in the flask culture the yields of gluconic acid were $83 \%$ and $56 \%$, respectively (data not shown). This indicates that xylose and cellobiose in the SM contributed to the production of gluconic acid and that the enzymatic hydrolysate from waste OA paper can be a good substitute carbon source for gluconic acid production in a culture of A. niger.

In the case of repeated batch production in flasks, the glucose consumption rate in SM was as same as that in GM (Figure 2a), although it become slower compared to that of GM after 72 h. Gluconic acid concentration reached $110 \mathrm{~g} / \mathrm{l}$ in both SM and GM over 72 hours. There was no difference between SM and GM until the third repetition of the batch culture. This result also strongly supports the claim that enzymatic hydrolysate does not have any negative effect on gluconic acid production and can be used as an alternative carbon source in the culture of $A$. niger. From the fourth run of the repeated batch culture, crystals formed in SM. These crystals are speculated as calcium gluconate, 
because concentrated gluconoc acid forms calcium gluconate in plenty of calcium ion. Both the crystals and the biomass bonded and formed large clumps, which might have hampered the oxygen transfer in A. niger.

\subsection{Gluconic acid production using SM in TBR}

In the repeated batch cultivation with an air supply, the average gluconic acid production rate was $6.16 \mathrm{~g} / \mathrm{l} . \mathrm{d}$ in SM (Figure 3b), while it was $24.3 \mathrm{~g} / \mathrm{l} . \mathrm{d}$ in GM (Figure 3d). Fresh media were exchanged four times over 27 days in SM (Figure 3a); in contrast, they were exchanged seven times over 22 days in GM (Figure 3c). The significant difference between the two media was the average glucose consumption rate: $11.0 \mathrm{~g} / \mathrm{l} . \mathrm{d}$ in SM, and $29.3 \mathrm{~g} / \mathrm{l} . \mathrm{d}$ in GM.

However, when the aeration was done with pure oxygen, the gluconic acid production rate increased to $23.7 \mathrm{~g} / \mathrm{l} . \mathrm{d}$ in SM (Figure 3b), and $35.0 \mathrm{~g} / \mathrm{l} . \mathrm{d}$ in GM (Figure 3d). Fresh media were exchanged six times over 15 days in SM (Figure 3a), while they were exchanged six times over 11 days in GM (Figure 3c). These results show that when SM was used in the culture of A. niger, oxygen transfer might have been limited. Supplying pure oxygen to SM holds great possibilities in the reutilization of waste paper resources.

The average gluconic acid yield based on glucose consumption was 60\% in SM and $67 \%$ in GM in the repeated batch culture using the TBR. No significant difference between yields was observed between batches until the fifth time the process was 
repeated. In addition, concentrations of the reducing sugars in SM showed no meaningful change throughout the cultivation period. The initial xylose concentration in SM was $39.4 \mathrm{~g} / \mathrm{l}$, but it decreased to $28.6 \mathrm{~g} / \mathrm{l} ; 72 \%$ of it remained. On the other hand, cellobiose concentrations were $6.7 \mathrm{~g} / \mathrm{l}$ and $2.0 \mathrm{~g} / \mathrm{l}$ before and after cultivation, respectively; only 29\% remained. Throughout the whole process, xylose consumption rate was low, 1.5 g/l.d. This is because $A$. niger preferentially consumes glucose as long as it exists in the SM. Cellobiose was consumed as quickly as glucose was; however, the total amount consumed was around 5\% that of glucose. Therefore, cellobiose had little effect on gluconic acid production compared to glucose. These results show that cellobiose and xylose contained in the SM were good carbon sources, but the amount of gluconic acid produced from them was negligible.

\subsection{Morphological change of A. niger in TBR}

Dry cell weight at a culture time of 15 days in SM was three times that of the GM at a culture time of 11 days: $66.88 \mathrm{~g} / \mathrm{l}$ in SM; $19.35 \mathrm{~g} / \mathrm{l}$ in the GM. This was similar in the flask cultures; dry cell weights in SM and GM were 51.5 g/l and 27.5 g/l, respectively. This may have been due to an uptake of cellulase remaining in SM as a nitrogen source. Pham (2003) reported that when 5\% of enzyme was used for the saccharification of waste paper the enzymatic hydrolysate contained $0.8 \mathrm{~g} / \mathrm{l}$ of protein, which contributed to the increase in the cellular yield of Rhizopus oryzae.

The increase in cellular yield might have caused a remarkable difference in the 
morphology of $A$. niger between SM and GM (Figure 4). In the culture using SM, the hyphae were thinner. Also, there were empty spaces between hyphae and many round-shaped clumps (Figure 4a). These clumps may have been complexes of calcium gluconate, calcium carbonate and fine cellulase crystals from the enzymatic hydrolysate. In contrast, when using GM, the hyphae were much thicker and they were in regular order (Figure 4b). Moreover, the rounded-shaped clumps observed in SM were hardly present in GM, but fine crystals, possibly calcium gluconate crystals were attached on hyphae as shown in Figure 4b.

\section{Discussion and Conclusions}

Much research on gluconic acid production has been done in cultures of $A$. niger using glucose as a carbon source, as shown in Table 1. Vassilev et al. (1993) immobilized A. niger on polyurethane foam and obtained $143 \mathrm{~g} / \mathrm{l}$ of gluconic acid from 150 g/l of reducing sugar from corn hydrolysate. Sankpal and Kulkarni (2002) immobilized A. niger on cellulose microfibers and obtained $6.56 \mathrm{~g} / \mathrm{l}$.h with $158 \mathrm{~g} / \mathrm{l}$ of gluconic acid in a continuous recirculation reactor. They also immobilized A. niger on cellulose and obtained $135 \mathrm{~g} / \mathrm{l}$ of gluconic acid during 61 days in surface culture (Sankpal et al., 1999), but utilization activity by mycelia decreased to $60 \%$ glucose conversion. Sakurai et al. (1989) have done repeated batch cultures of A. niger immobilized on nonwoven fabric, and they obtained $300 \mathrm{~g} / \mathrm{l}$ of gluconic acid with 60g/l.h volumetric productivity. These studies on gluconic acid production were 
performed using glucose or corn hydrolysate. This paper represents the first experiment using enzymatic hydrolysate of waste paper for gluconic acid production. Gluconic acid yield based on glucose consumption in flasks using SM was 92\%, and 80\% in GM. Although the values gradually decreased as the process was repeated, the average gluconic acid yield in SM did not show any significant difference from that in GM. The gluconic acid productivity was lower compared to those of immobilized $A$. niger using glucose as carbon source, but 1.13 g/l.h gluconic acid productivity with 80-100 g/l gluconic acid was achieved. This study may be the first step in utilizing hydrolysate of waste paper to produce gluconic acid.

The production of gluconic acid by A. niger is dependent on oxygen transfer rate since the production process is a simple oxidation of glucose. To increase oxygen transfer, Sakurai et al. (1989) maintained dissolved oxygen at 150 ppm by supplying high pressure pure oxygen at 6 atm. Klein et al. (2002) used an air-lift bioreactor to increase oxygen transfer rate and obtained a production rate of $2.3 \mathrm{~g} / \mathrm{l}$.h with $150 \mathrm{~g} / \mathrm{l}$ gluconic acid. To obtain a high gluconic acid productivity a sufficient level of oxygen supply is indispensable. However, in the present study the dissolved oxygen concentration could not be measured during cultivations, because the membrane of the dissolved oxygen sensor was clogged by hyphae of A. niger. Higher productivity may have been obtained if a sufficient level of oxygen were supplied.

It has been reported previously that L-(+)-lactic acid in the culture of Rhizopus oryzae using enzymatic hydrolysate of waste paper was approximately $35 \%$ less than that in GM (Park et al., 2004). However, in this study, the enzymatic hydrolysate 
composed of reducing sugars, such as xylose, cellobiose and other sugars, did not show any negative influence on the production of gluconic acid. This presents a great possibility to reutilize waste paper in an alternative way.

However, the morphology of A. niger in SM was different from that in GM: in SM, it was larger and fluffier than that in GM as observed with the scanning electronic microscope. In fact, the dry cell weights in the SM were 2 and 3.5 times those in GM in the flasks and the TBR, respectively. This may have been due to an uptake of cellulase from the enzymatic hydrolysate. This clumpy morphology in SM may have caused oxygen depletion in the reactor and ultimately a decrease in gluconic acid yield.

The production of gluconic acid from enzymatic hydrolysate of waste paper may be a waste paper recycling alternative, but the process needs improvement. It may be possible to establish an effective process of gluconic acid production, such as the culture of $A$. niger and simultaneous recovery of gluconic acid. Since the demand to reutilize waste paper will increase in the near future due to environmental concerns such research is necessary to provide alternative recycling methods.

\section{References}

Klein, J., Rosenberg, M., Markos, J., Dolgos, O., Kroslak, M., Kristofikva, L., 2002. Biotransformation of glucose to gluconic acid by Aspergillus niger - study of mass transfer in an air lift bioreactor. Biochem. Eng. J. 10, 197-205.

Park, E.Y., Ikeda, Y., Okuda, N., 2002. Empirical evaluation of cellulase on enzymatic 
hydrolysis of waste OA paper. Biotechnol. Bioprocess. Eng. 7, 1-6.

Park, E.Y., Michinaka, A., Okuda, N., 2001. Enzyme hydrolysis of waste office paper using viscosity operating parameter. Biotechnol. Prog. 17, 379-382.

Park, E.Y., Pham, N.A., Okuda N., 2004. Bioconversion of waste office paper to L-(+)-lactic acid by the filamentous fungus Rhizopus oryzae. Bioresource Technol. 93, 77-83.

Pham, N.A., 2003. Study on L(+)-lactic acid production from Rhizopus oryzae using enzyme hydrolysate of waste paper. Masters thesis, Shizuoka University (Japanese).

Sakurai, H., Lee, H.W., Sato, S., Mukataka, S., Takahashi, J., 1989. Gluconic acid production at high concentrations by Aspergillus niger immobilized on a nonwoven fabric. J. Ferment. Bioeng. 67, 6, 404-408.

Sankpal, N.V., Joshi, A.P., Sutar, I.I., Kulkarni, B.D., 1999. Continuous production of gluconic acid by Aspergillus niger immobilized on cellulosic support: study of low pH fermentative behavior of Aspergillus niger. Process Biochem. 35, 317-325.

Sankpal, N.V., Kulkarni, B.D., 2002. Optimization of fermentation conditions for gluconic acid production using Aspergillus niger immobilized on cellulase microfibrils. Process Biochem. 37, 1343-1350.

Scott, C.D., Davison, B.H., Scott, T.C., Woodward, J., Dees, C., Rothrock, D.S., 1994. An advanced bioprocessing concept for the conversion of waste paper to ethanol. Appl. Biochem. Biotechnol. 45/46, 641-653.

Terasawa, M., 2005. Paper recycling promotion center. Paper recycling handbook 2005. Wayman, M., Chen, S., Doan, K., 1993. Production of fuel ethanol from the waste paper 
in garbage. Trans. IChemE B 71, 141-143.

Vassilev, N.B., Vassileva, M.Ch., Spassova, D.I., 1993. Production of gluconic acid by Aspergillus niger immobilized on polyurethane foam. Appl. Microbiol. Biotechnol. 39, 285-288. 
Table 1

Summary of gluconic acid production in cultures of $A$. niger

\begin{tabular}{|c|c|c|c|c|}
\hline Carbon source & Culture method & $\begin{array}{l}\text { Gluconic acid } \\
\qquad(\mathrm{g} / \mathrm{l})\end{array}$ & $\begin{array}{c}\text { Volumetric } \\
\text { productivity (g/l.h) }\end{array}$ & References \\
\hline $\begin{array}{l}\text { Reducing sugar from corn } \\
\text { hydrolysate }\end{array}$ & Repeated batch culture ${ }^{\mathrm{a}}$, polyurethane foam ${ }^{\mathrm{b}}$ & 143 & 1.44 g/g support.h & $\begin{array}{l}\text { Vassilev et al. } \\
\text { (1993) }\end{array}$ \\
\hline Glucose & Continuous surface culture $^{\mathrm{a}}$, cellulose ${ }^{\mathrm{b}}$ & 135 & 11.3 & Sankpal et al. (1999) \\
\hline Glucose & Repeated batch culture ${ }^{\mathrm{a}}$, nonwoven fabric ${ }^{\mathrm{b}}$ & 300 & 60 & Sakurai et al. (1989) \\
\hline Glucose & $\begin{array}{l}\text { Continuous recirculation reactor }{ }^{\mathrm{a}} \text {, cellulose } \\
\text { microfibril }^{\mathrm{b}}\end{array}$ & 158 & 6.56 & $\begin{array}{l}\text { Sankpal and } \\
\text { Kulkarni (2002) }\end{array}$ \\
\hline Glucose & Batch culture in air-lift bioreactor ${ }^{\mathrm{a}}$ & 150 & 2.3 & Klein et al. (2002) \\
\hline $\begin{array}{l}\text { Enzymatic hydrolysate of } \\
\text { waste paper }\end{array}$ & $\begin{array}{l}\text { Repeated batch culture in turbine blade } \\
\text { reactor }^{\mathrm{a}} \text {, containment in stainless mesh } \\
\text { cylinder }^{\mathrm{b}}\end{array}$ & $80-100$ & 1.13 & This work \\
\hline
\end{tabular}




\section{Figure Captions}

Fig. 1. (a) Concentrations of glucose (circles) and gluconic acid (squares) in the batch culture of A. niger using the SM (closed) and GM (open). (b) Concentrations of xylose (closed triangles) and cellobiose (closed circles) in SM.

Fig. 2. Time course of glucose consumption (a) and gluconic acid production (b) in the repeated batch culture using the SM (closed) and the GM (open). Arrows indicate medium exchange time.

Fig. 3. Time courses of repeated batch cultures using TBR using the SM (a, b) and in the GM (c, d). (a, c) Glucose concentration. (b, d) Gluconic acid production. Two different aerations were carried out: air supply (open); pure oxygen supply (closed).

Fig. 4. Morphology of A. niger by SEM (a) in SM at a culture time of $15 \mathrm{~d}$ and (b) in GM at $11 \mathrm{~d}$. Both biomass samples were taken from the bottom of the culture space in the TBR after finishing the cultivations with pure oxygen supply. The arrows in (a) and (b) show a complex of fine cellulose, gluconate and calcium carbonate and crystals of calcium gluconate, respectively. 
Fig. 1, Ikeda et al.

a)

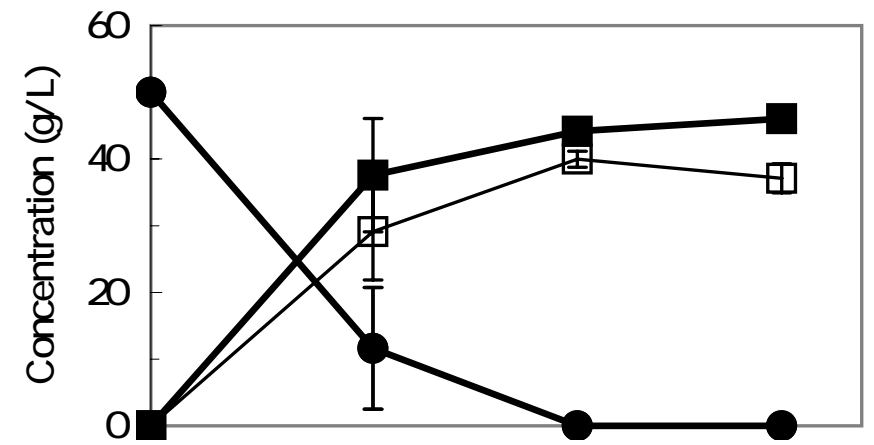

b)

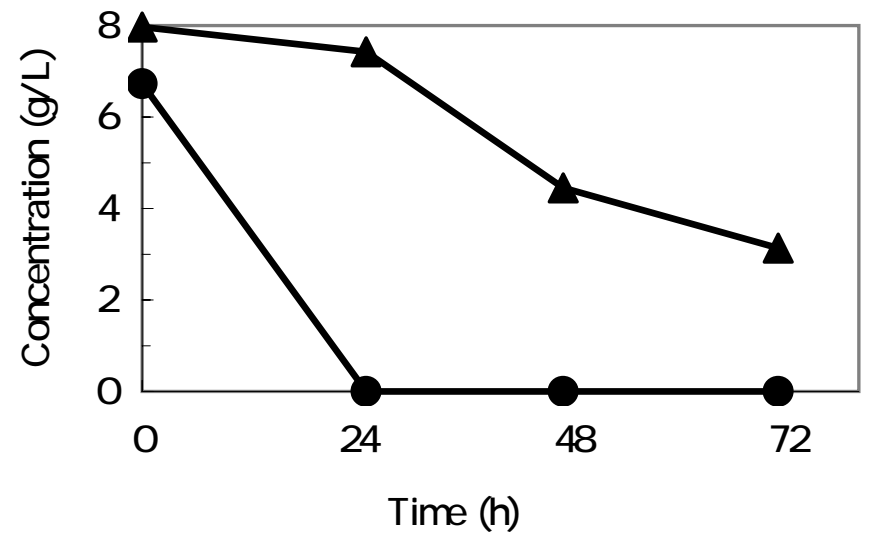


Fig. 2. Ikeda etal.

a)

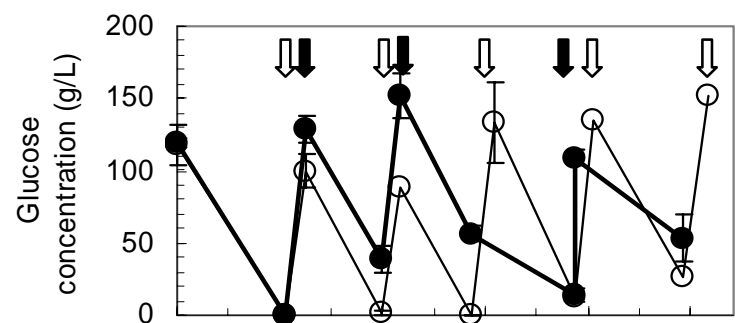

b)

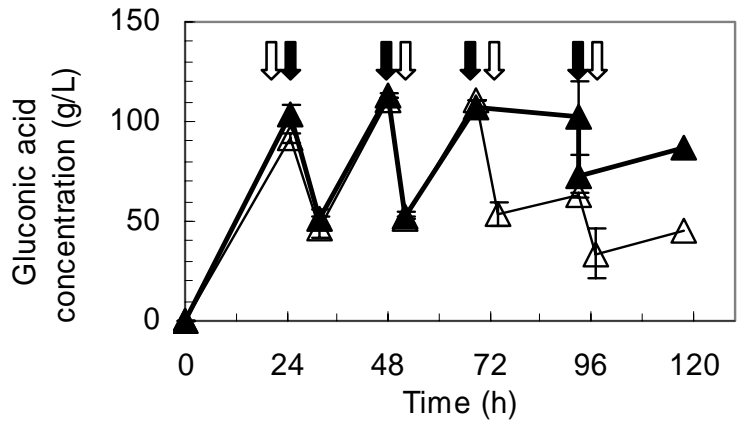


a)

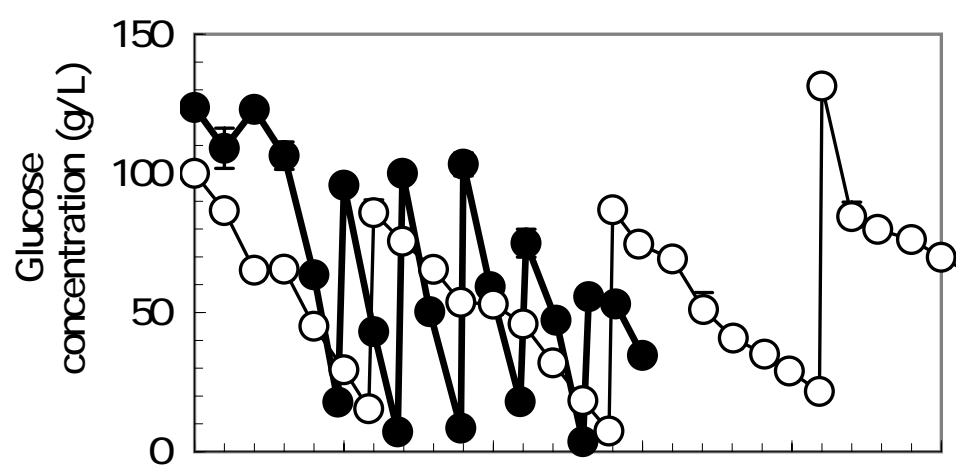

b)

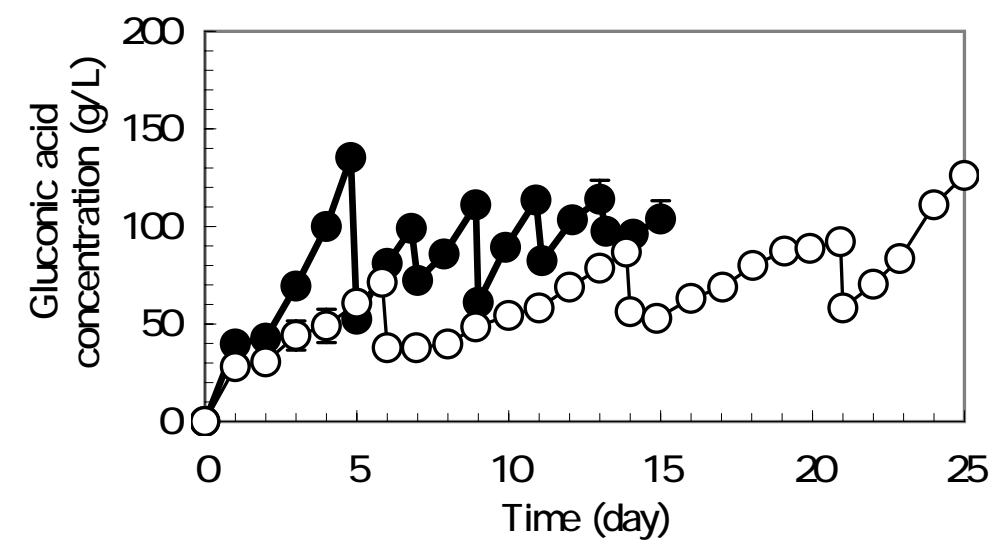

c)
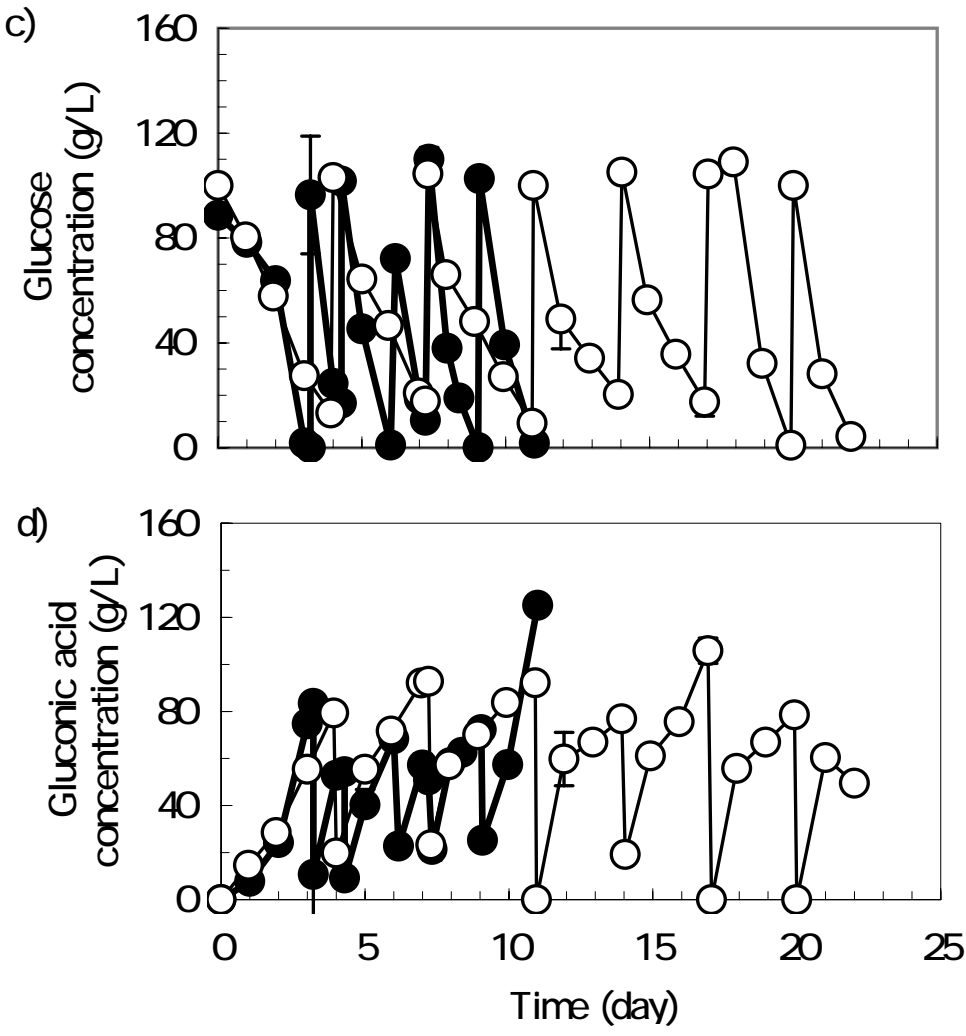
Fig. 4, Ikeda et al.
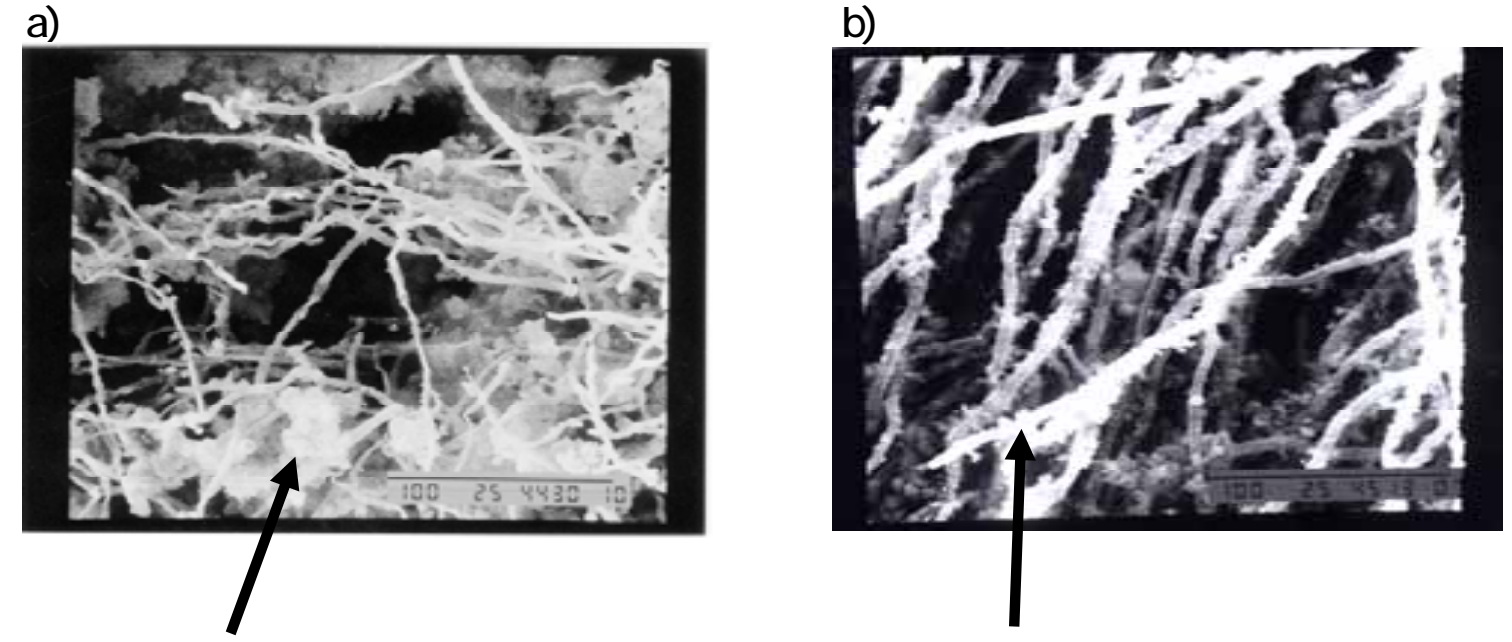